The authors strongly recommend that vegetation length be measured by transesophageal echocardiography upon initial diagnosis of IE; longer vegetations should be treated as highrisk factors. Further prospective studies are needed to determine whether such patients should undergo early surgery.

Pippa Murdie

Original article Thuny F et al. (2005) Risk of embolism and death in infective endocarditis: prognostic value of echocardiography—a prospective multicenter study. Circulation 112: 69-75

\section{Importance of local C-reactive protein release}

Researchers in Japan recently reported results of a two-protocol study that assessed the local release of C-reactive protein (CRP) from both atherosclerotic plaques and coronary vessel walls subjected to stenting.

The first protocol involved the measurement of serum high-sensitivity CRP (hsCRP) levels in blood samples taken from the coronary arteries of 36 patients with stable angina and 13 patients with unstable angina, all of whom were undergoing percutaneous coronary intervention for isolated culprit lesions. Samples were taken from areas both distal and proximal to culprit lesions. The second protocol involved the measurement of serum hsCRP levels and activated leukocyte integrin MAC-1 in peripheral and coronary sinus blood taken from 20 patients with stable angina who underwent coronary stent implantation.

In the first protocol, the distal CRP level was higher than the proximal CRP level in both groups of patients, creating a translesional CRP gradient. Proximal CRP was also found to correlate with translesional CRP $(P<0.001)$ in all patients. The second protocol revealed a timedependent increase in both plasma CRP levels and the transcardiac CRP gradient following coronary stenting, reaching a peak at $48 \mathrm{~h}$.

The authors conclude that the presence of translesional or transcardiac CRP gradients suggests that at least a proportion of serum CRP is produced at the lesion site, and therefore evaluation of local CRP may allow recognition of plaque instability.

Christine Kyme

Original article Inoue T et al. (2005) Local release of Creactive protein from vulnerable plaque or coronary arterial wall injured by stenting. J Am Coll Cardiol 46: 239-245

\section{Risk factors for gastrointestinal complications after cardiac surgery}

Though gastrointestinal complications in patients who have undergone heart operations are rare, they pose a significant mortality and morbidity risk. Mangi and colleagues therefore investigated the variables that could cause gastrointestinal complications after cardiac surgery, to enable strategies for alleviating this risk to be identified.

Out of 8,709 patients who underwent a heart operation at the Massachusetts General Hospital between 1997 and 2003, 46 subsequently suffered from gastrointestinal complications and were therefore included in this study. The authors identified 89 preoperative, intraoperative and postoperative variables of gastrointestinal complications, which they compared against a control group of 250 randomly selected patients.

The results demonstrated the most frequent gastrointestinal complication after cardiac surgery to be mesenteric ischemia, which was suspected or documented in 31 out of the 46 patients, proving fatal in $68 \%$ of these cases. Preoperative predictors of mesenteric ischemia include atheroembolization, heparin-induced thrombocytopenia and hypoperfusion. Other gastrointestinal complications included diverticulitis, pancreatitis, peptic ulcer disease and cholecystitis. Significant predictors of death from a gastrointestinal complication after cardiac surgery included New York Heart Association class II and IV heart failure, smoking, chronic obstructive pulmonary disease and syncope at time of presentation.

The authors conclude that early recognition is important for survival from mesenteric ischemia as this complication often occurs within hours after surgery. They suggest that this could be achieved by fast-track extubation pathways or minimum sedation. Ultimately, techniques to reduce the occurrence of mesenteric ischemia after heart surgery will be necessary to reduce overall mortality.

Marie Lofthouse

Original article Mangi AA et al. (2005) Gastrointestinal complications in patients undergoing heart operation. An analysis of 8709 consecutive cardiac surgical patients. Ann Surg 241: 895-904 